\title{
Biochemical Characterization of Acetamiprid Resistance in Laboratory-Bred Population of Aedes aegypti L. Larvae
}

\author{
Roopa Rani Samal $^{1 *}$, Kungreiliu Panmei ${ }^{1}$, P Lanbiliu ${ }^{1}$, Sarita Kumar ${ }^{1}$ \\ ${ }^{I}$ Department of Zoology, Acharya Narendra Dev College, University of Delhi, Kalkaji, New Delhi 110019, India \\ *Corresponding author. Email: rupasamal29@gmail.com
}

\begin{abstract}
The constant rise in cases of Zika, Dengue and Chikungunya worldwide has made control of Aedes aegypti a principal concern. The most recommended plan to control mosquito-borne diseases primarily lies on vector management and disturbing their disease-transmission cycle. Wide-ranging use of different classes of organic insecticides for mosquito control has led to the development of high levels of resistance making them less operative at safe dosages imposing us to explore novel insecticides. Present study investigates the bioefficacy of a neonicotinoid, acetamiprid on the Ae. aegypti larvae, development of resistance after subjecting acetamiprid selection pressure for 10 successive generations and biochemical characterization of the resistance developed. Acetamiprid exposure of the parent population of Ae. aegypti early fourth instars resulted in respective $\mathrm{LC}_{50}$ and $\mathrm{LC}_{90}$ values of $0.188 \mathrm{ppm}$ and $1.315 \mathrm{ppm}$. Selection with acetamiprid for 10 successive generations (ACSF-10) reduced its efficacy by 20 -fold. Involvement of four enzymes; alphaesterases, beta-esterases, glutathione-S-transferases and acetylcholinesterases in development of acetamiprid resistance was investigated to uncover mode of action of acetamiprid. An elevation of 1.4-fold and 2.1-fold was observed in alpha-esterases and beta-esterases activity in ACSF-10 as compared to ACSF-5. However, activity of glutathione-S-transferases decreased in ACSF-5 which rose to 12-fold in ACSF-10. Similarly, the activity of acetylcholinesterases was found to be much higher in resistant generations as compared to the parental strains. The results indicated individual/synergistic contribution of different enzymes leading to acetamiprid detoxification. Further research is being conducted to identify the role of target site mutations in resistance development.
\end{abstract}

Keywords: Aedes aegypti, acetamiprid, esterases, glutathione-S-transferase, acetylcholinesterases

\section{INTRODUCTION}

The WHO global strategy for dengue prevention and control by 2020 is based on the fact that almost half of the world population resides in dengue-prone area; resulting in 50 to 100 million estimated annual dengue infections. Currently, approximately $75 \%$ population of Asia-Pacific region is exposed to dengue. The actual number of cases of dengue is even worse, because of severe underreporting and misclassification [1, 2]. In addition, most countries in Southeast Asia with endemic malaria are experiencing increased Aedes-borne diseases due to ecological changes arising from poorly controlled population movement and extensive exploitation of natural environments.

The prevention of dengue transmission primarily depends on reduction of the human-vector contact using residual chemical compounds; while dengue infection can be kept under check by accurate diagnosis and prompt effective treatment $[3,4]$. For decades, DDT was used in mosquito vector control programs as an interdomiciliary spray; which was gradually discontinued because of social and environmental concerns. The organochlorines were then replaced with organophosphates followed by synthetic pyrethroids. Since then, pyrethroids have been used extensively for the insecticide treatment of bed nets (ITN) and as indoor residual sprays (IRS) in many parts of the country. However, repeated contact with these insecticides has led, in some cases, to high levels of resistance in vector populations. The increased development of mosquito resistance to pyrethroids is of particular concern for many integrated mosquito control programs that utilize insecticides for vector control [5].

Despite extensive research in the field, management of insecticide resistance remains the major challenge in mosquito control leading to the need of developing strategies which could ensure long-term efficacy of toxicants and; delay or prevent the development of resistance. Identification and introduction of new insecticides in the fields with mosquito control potential and novel mode of action has become a major task in front of the researchers. Neonicotinoids are among such 
insecticides which are being explored by the researchers as mosquito control agents. These are the synthetic analogues of natural insecticide nicotine, an active component of tobacco and are considered agonist neurotoxicants targeting various sensitive sites in insect nervous system [6-8]. Initially discovered in late 1980s, these are now used for various purposes ranging from plant protection, biocides, and veterinary products to the control of invertebrate pests in fish farming [9]. Reports reveal high pest control efficacy of neonicotinoids as they have been effective at much lower concentration and quantity in comparison to the traditionally used insecticides [10]. In addition, their low mammalian toxicity, negligible cross resistance and unique mode of action suggest the possible use of neonicotinoids as ideal alternatives to various synthetic organic insecticides [11]. The newest major group of neonicotinoids includes Acetamiprid, Imidacloprid, Clothianidin, Dinotefuran, Nitenpyram, Thiacloprid and Thiamethoxam. The halflife and residual nature of these compounds vary widely from a few months to much longer durations [12-14]. These chemicals have been reported effective against a variety of economically important crop pests including whitefly, aphids, leafhoppers, wireworms, plant hoppers and mealybugs [15] and also showed efficacy against mosquito larvae as compared to other classes [16].

Acetamiprid, an odourless neonicotinoid insecticide manufactured under the trade names Assail, and Chipco by Aventis CropScience India, is a nicotinic agonist that reacts with nicotinic acetylcholine receptors (nAChRs) [17] located in the post-synaptic neural dendrites of brain, spinal cord, ganglia and muscular junctions; and mediates fast cholinergic transmission, a unique mode of action $[18,19]$. Acetamiprid is also known to possess both acute contact and stomach poisoning activity [18, 20]. This insecticide, therefore, has become an important component of integrated pest management since it was registered commercially in 1995.

In this study, the larvae of Ae. aegypti were exposed to acetamiprid for 10 successive generations to assess the probable larval resistance development. Thereafter, we conducted a series of biochemical assays integrated with dose-mortality bioassays for detection of resistance and to outline the underlined mechanisms involved in neonicotinoid resistance in Ae. aegypti $\mathrm{L}$.

\subsection{Materials and Methods}

\subsubsection{Culture of Aedes aegypti $L$.}

Dengue fever mosquitoes, Ae. aegypti, at adult and larval stages, were obtained from ICGEB (International Centre for Genetic Engineering and Biotechnology), New Delhi, India. The colony was maintained in an insect rearing unit of Insect Pest and Vector Laboratory, Acharya Narendra Dev College, University of Delhi, India under controlled conditions of $28{ }^{\circ} \mathrm{C} \pm 1{ }^{\circ} \mathrm{C}, 80 \% \pm 5 \% \mathrm{RH}, 14$ hr of light and $10 \mathrm{hr}$ of darkness [21]. Adults kept in clothed cages were fed on sugary juice of water-soaked raisins while female Ae. aegypti were provided with occasional blood meals for egg maturation. Eggs collected in an ovitrap were transferred into the enamel trays filled with at least 1.5-2.0 L of dechlorinated water. The hatched larvae were fed on powdered dog biscuits and yeast in a ratio of 3:1 till they transformed into pupae [22, 23]. Trays were kept clean and water was changed every day to prevent formation of scum on the water surface. The pupae collected on regular basis were kept in clothed cages for adult emergence.

\subsubsection{Insecticide susceptibility test}

The parent strain (PS) of Ae. aegypti L. was exposed to acetamiprid to estimate its larvicidal efficacy [22, 23]. The larvae were then subjected to selection pressure of acetamiprid at $\mathrm{LC}_{90}$ level till 10 successive generations to assess the development of resistance. The acetamipridselected $5^{\text {th }}$ generation (ACSF-5) and $10^{\text {th }}$ generation (ACSF-10) were employed in the current experiments.

\subsubsection{Biochemical assay}

The larvae of parent generation of Aedes aegypti L. and those obtained after subjection to selection pressure with acetamiprid for successive 5 and 10 generations were characterized biochemically. The standard method to identify the probable biochemical mechanism involved in insecticides resistance provided by WHO [24] was used with few minor changes. Individual larva was homogenized in $200 \mu \mathrm{L}$ of autoclaved water on ice. The $25 \mu 1 \times 2$ (replicate) of homogenized mixture was segregated for the acetylcholinesterase assay and the remaining homogenate was spun in a refrigerated microfuge at maximum speed for $30 \mathrm{~s}$. Supernatant separated after centrifugation was used for estimation of proteins, alpha-esterases, beta-esterases and glutathione-stransferases.

\subsubsection{Protein estimation}

The $10 \mu \mathrm{L} \times 2$ (replicate) supernatant from all the larval strains was pipetted in the microtiter plate. The $300 \mu \mathrm{L}$ of BIORAD protein reagent was added to the homogenate. A blank was run simultaneously replacing homogenate with the water. The absorbance of the mixture was read at $570 \mathrm{~nm}$ with the help of ELISA plate reader. The standard curve was plotted and the concentration of the protein of individual larvae from PS, ACSF-5 and ACSF-10 was calculated in $\mathrm{mg} / \mathrm{mL}$ and in $\mathrm{n}$ moles. 


\subsubsection{Non-specific esterases estimation}

The $10 \mu \mathrm{L} \times 2$ (replicate) of the supernatant of each strain was pipetted in the microtiter plate. The $200 \mu \mathrm{L}$ of Alpha/Beta naphthyl acetate was added in each titer and incubated for 15 mins. Visual changes were interpreted by adding $50 \mu 1$ of fast blue stain (freshly prepared) in each well. The absorbance was measured at $570 \mathrm{~nm}$ to evaluate the elevation in the level of esterases in each strain.

\subsubsection{Glutathione-S-transferases bioassay}

The $20 \mu \mathrm{L} \times 2$ (replicate) of the supernatant of each strain was pipetted in the microtiter plate to which $50 \mu \mathrm{L}$ of $2 \mathrm{mM} \mathrm{GSH}$ (Reduced glutathione) and $50 \mu \mathrm{l}$ of $1 \mathrm{mM}$ CDNB (1-Chloro-2,4-dinitrobenzene) was added. The ELISA plates were then read continuously for $5 \mathrm{mins}$ at $340 \mathrm{~nm}$ to study the enzyme kinetics.

\subsubsection{Acetylcholinesterase's inhibition bioassay}

The $25 \mu \mathrm{L} \times 2$ (replicate) of crude insect homogenate of each strain was placed in separate wells of a microtiter plate. The ASCHI (Acetylthiocholine iodide) solution was divided into two $10 \mathrm{~mL}$ aliquots. One aliquot was added with $20 \mu \mathrm{L}$ of $0.1 \mathrm{M}$ propoxur. The $145 \mu \mathrm{L}$ of $1 \%$ solution of Triton X-100 was added to the homogenate to solubilize acetylcholinesterases, followed by addition of $10 \mu \mathrm{L}$ of DTNB (Dithiobis 2-nitrobenzoic acid) solution. Thereafter, $25 \mu \mathrm{L}$ of ASCHI was added to one replicate and while $25 \mu \mathrm{l}$ of ASCHI+propoxur was added to the other replicate. The reaction mixture was then incubated for $1 \mathrm{hr}$ after which end point reading was taken by reading the absorbance at $405 \mathrm{~nm}$.

\subsection{Our Contribution}

This paper discusses the possible use of acetamiprid for the control of dengue vector, Ae. aegypti. The development of acetamiprid resistance in the vector and characterization of the underlying biochemical and molecular mechanisms that may potentially play a role in the resistance are elucidated. The variations in the enzyme levels in the resistant Ae. aegypti population are determined while the role of target site mutations in resistance development is under exploration. These investigations could help in formulation of resistance management strategies to eliminate heterozygotes and resistant homozygotes, the efficacy of which can only be established after field trials.

\subsection{Paper Structure}

The rest of the paper is organized as follows. Section 2 explains the insecticide resistance, its mechanism the biochemical characterization of resistance. The acetamiprid susceptibility in Ae. aegypti and development of resistance are presented and analysed. This section tells about the correlation of three major classes of detoxifying enzymes: Non-specific esterases, Glutathione-S-transferases and acetylcholinesterase with increase in level of acetamiprid resistance in Ae. aegypti. These results are discussed with that conducted elsewhere. Finally, Section 3 concludes the paper and presents direction for future research.

\section{RESULTS AND DISCUSSION}

Employment of chemical insecticides is the prime strategy in control of mosquito vectors. Resistance to different classes of insecticides; organochlorines, organophosphates and pyrethroids has been reported in different mosquito vectors which may be attributed to constant selection pressure of insecticide used in agriculture as well as in residential areas [25-27]. The forced paths available for the supervision of insecticide resistance have created an urgent necessity to identify novel insecticides for effective control of mosquito species which have developed multiple resistances to the insecticides belonging to different classes [28]. Present study involves, neonicotinoids, a class of neurotoxic insecticides with highly systemic toxicity and long-term persistence. They bind to the nicotinic acetylcholine receptors in the nervous system and block the route of nerve impulses, the binding potency conferred by a particular confirmation of the molecule [29].

The insecticidal potential of different neonicotinoids has been reported earlier [28, 30]. Most of these studies are focused on the toxicity assays of imidacloprid. In the present study, an attempt was made to study the probable use of a neonicotinoid, acetamiprid, for Ae. aegypti management. The early fourth instars of Ae. aegypti were tested for their susceptibility and tolerance levels against the insecticide. The parent strains and those subjected to continuous selection pressure of acetamiprid (ACSF-5 and ACSF-10) were assessed for elevation in the xenobiotic metabolizing enzymes. The study displayed high susceptibility of the Ae. aegypti larvae to the acetamiprid. Exposure of the early fourth instars of parent generation resulted in $\mathrm{LC}_{50}$ and $\mathrm{LC}_{90}$ values of $0.188 \mathrm{ppm}$ and 1.31 ppm, respectively. Selection of these larvae with acetamiprid at $\mathrm{LC}_{90}$ levels resulted in the development of considerable level of resistance. The early fourth instars of Ae. aegypti subjected to continuous selection pressure of acetamiprid for 10 successive generation developed 19.74fold resistance to acetamiprid (Table 1). The respective $\mathrm{LC}_{50}$ and $\mathrm{LC}_{90}$ values recorded in the parent strain rose to 3.711 and $10.088 \mathrm{ppm}$ in ACSF-10. Similar results were reported by Uragayala et al. [28] in an Indian strain of Ae. aegypti susceptible to organochlorines, organophosphates and pyrethroids. They obtained a little higher $\mathrm{LC}_{50}$ value $(0.558 \mathrm{ppm})$ on larval bioassay with imidacloprid. 
Table 1 Larval $\mathrm{LC}_{50}$ and $\mathrm{LC}_{90}$ (in ppm) of parent susceptible (PS) and acetamiprid-larval selected strain of Aedes aegypti selected for 10 successive generations

\begin{tabular}{|c|c|c|c|c|c|}
\hline Strain & $\mathbf{L C}_{\mathbf{5 0}}(\mathbf{p p m})$ & $\mathbf{L C}_{\mathbf{9 0}}(\mathbf{p p m})$ & Heterogeneity $\boldsymbol{\chi}^{\mathbf{2}}(\mathbf{D f})$ & Slope \pm SEM & RR \\
\hline PS & $\begin{array}{c}0.188 \\
(0.119-0.248)^{* *}\end{array}$ & $\begin{array}{c}1.315 \\
(0.759-5.241)^{* *}\end{array}$ & $3.395(3)$ & $1.517 \pm 0.295$ & ---- \\
\hline ACSF-5* & $\begin{array}{c}1.659 \\
(0.727-2.065)\end{array}$ & $\begin{array}{c}4.509 \\
(3.284-20.236)\end{array}$ & $2.285(3)$ & $2.951 \pm 0.146$ & 3.427 \\
\hline ACSF-10 & $\begin{array}{c}3.711 \\
(2.915-4.356)\end{array}$ & $\begin{array}{c}10.088 \\
(7.120-32.184)\end{array}$ & $0.959(6)$ & $2.975 \pm 0.119$ & 19.74 \\
\hline
\end{tabular}

ACSF* - Acetamiprid selected filial RR- Resistance Ratio

**Figures in parentheses represent lower and upper 95\% Confidence Limit

$\mathrm{LC}_{50}$ - Lethal Concentration at which 50\% larvae are killed, $\mathrm{LC}_{90}$ - Lethal Concentration at which 90\% larvae are killed; SEM=Standard Error of Mean, $\mathrm{Df}=$ degree of freedom

Identification of biochemical-based resistance mechanisms using microplate enzyme assays in a single mosquito is more informative and could be of value in early detection of insecticide resistance in field population [13]. Target site insensitive AChE assay and detoxification enzymes, aand $\beta$-esterases and GST assays were conducted to identify the probable biochemical mechanism involved in acetamiprid resistance.

The larval selection with acetamiprid for 10 successive generations resulted in accountable biochemical alterations in of Ae. aegypti larvae. Alpha-esterase activity increased by 1.27 -fold in ACSF-10 as compared to the susceptible generation. However, the results showed an insignificantly reduced esterase activity by 0.93 -fold in ACSF-5 as compared to the parent strain (PS) (Table 2; Figure 1). The elevated levels of beta-esterase activity in Ae. aegypti was evidenced by the appearance of pink colour in the solution indicating the formation of 2naphthol.

Beta-esterase activity oscillated a lot between the generations tested. Subjection of acetamiprid selection pressure for 5 generations decreased beta-esterase activity by 0.63 -fold which later increased significantly by 1.33 - fold in ACSF-10 (Table 2). The esterase activity in parent strain and ACSF-5 did not differ significantly $(p>0.05)$, whereas the activity was statistically different from that observed in ACSF-10 (Figure 2). Similar results were reported by Hemingway [27] who found quantitative increase of esterases in malathion-resistant An. stephensi from Pakistan.

The Glutathione-S-transferase assay is based on the conjugation of reduced glutathione with CDNB (3-(2chloro-4-nitrophen ('1)-glutathione) in presence of Glutathione-S-transferase as visual interpretation is not possible due to lack of any colour appearance in this reaction. The conjugation is calculated by noting the enzyme kinetics through scanning the activity continuously for at least 5 mins, which is then calculated in nmoles $/ \mathrm{min} / \mathrm{mL}$. The results showed that ACSF-5 exhibited significantly reduced GST activity as compared to PS $(p<0.05)$. However, the activity shot up in ACSF-10 by 1.50 (Table 2; Figure 3). In addition, an increased AChE activity was observed in the average population of ACSF-5 and ACSF-10; resulting in $0.07 \%$ decreased AChE inhibition in ACSF-5 which further decreased to $2.94 \%$ in ACSF-10. Safi et al. [31] also reported metabolic-based

Table 2 Mean Protein, $\alpha$ - and $\beta$-esterase, GST activities and \% AChE inhibition in laboratory-bred population of Aedes aegypti L. after exposure to acetamiprid for 10 successive generations.

\begin{tabular}{|c|c|c|c|c|c|}
\hline Strain & $\begin{array}{c}\text { Protein Concentration } \\
\text { (nmoles) } \pm \\
\text { SEM* }\end{array}$ & $\begin{array}{c}\alpha \text {-Esterase } \\
(\text { nmoles/min } / \mathbf{m g} \\
\text { protein }) \pm \text { SEM* }\end{array}$ & $\begin{array}{c}\beta \text {-Esterase } \\
(\text { nmoles} / \mathrm{min} / \mathrm{mg} \\
\text { protein }) \pm \mathrm{SEM} *\end{array}$ & $\begin{array}{c}\text { GST activity } \\
(\mathrm{nmoles} / \mathrm{min} / \mathrm{ml}) \\
\pm \text { SEM* }\end{array}$ & $\begin{array}{c}\% \text { AChE } \\
\text { inhibition } \pm \\
\text { SEM* }\end{array}$ \\
\hline PS & $8.06 \pm 0.27 \mathrm{a}$ & $1.32 \pm 0.05 \mathrm{a}_{1}$ & $2.23 \pm 0.22 \mathrm{a}_{2}$ & $1.80 \pm 0.18 b_{3}$ & $62.00 \pm 2.95 \mathrm{a}_{4}$ \\
\hline ACSF-5 & $7.95 \pm 0.27 \mathrm{a}$ & $1.29 \pm 0.03 \mathrm{a}_{1}$ & $1.40 \pm 0.42 \mathrm{a}_{2}$ & $0.23 \pm 0.02 c_{3}$ & $61.93 \pm 2.33 \mathrm{a}_{4}$ \\
\hline ACSF-10 & $8.30 \pm 0.29 a$ & $1.77 \pm 0.05 b_{1}$ & $2.98 \pm 0.17 b_{2}$ & $2.71 \pm 0.19 \mathrm{a}_{3}$ & $59.06 \pm 4.12 \mathrm{a}_{4}$ \\
\hline
\end{tabular}

*Figures in each column followed by different letters are significantly different $\mathrm{p}<0.05$, one-way ANOVA followed by Tukey's all pair wise multiple Comparison test; SEM: Standard error of Mean 
resistance development in Ae. aegypti. These are well known and established mechanisms for resistance to pyrethroids and OPs in mosquitoes [26]. The involvement of P450 mediated monooxygenases, elevated nonspecific esterases, and reduced sensitivity of sodium ion channels in insects has been reported as common insecticide resistance strategy against pyrethroids [3235]. Moreover, increased level of glutathione-Stransferases (GSTs) has been associated with conferring pyrethroid inhibition in different mosquito species; $A e$. aegypti, An. gambiae and An. dirus B [36].

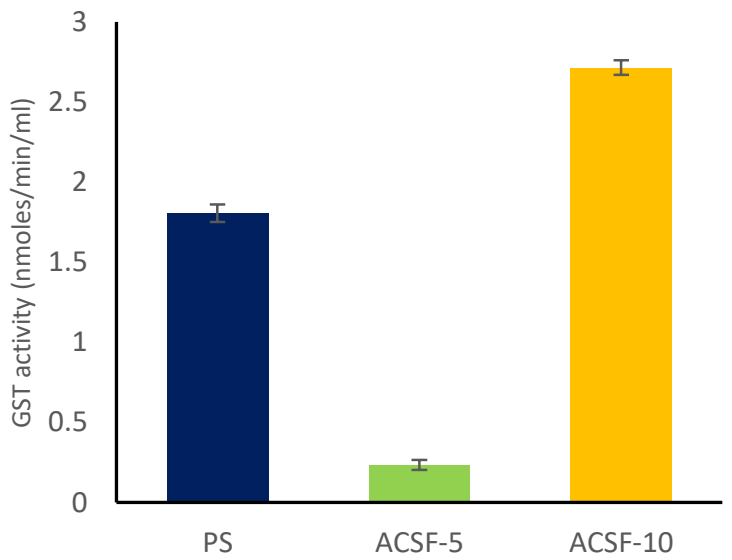

Figure 3 Glutathione-S-transferase activity in different strains of Ae. aegypti larvae

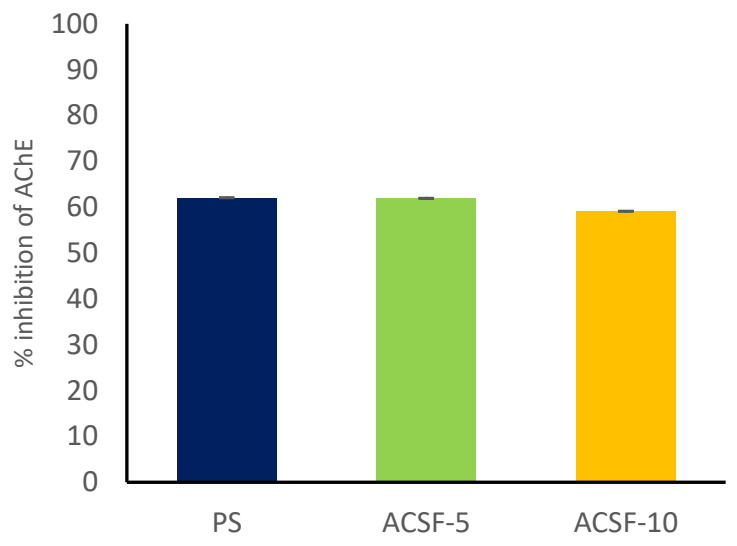

Figure 4 Inhibition of acetylcholinesterase in different strains of Ae. aegypti larvae
Figure 2 Beta-esterases activity in different strains of Ae. aegypti larvae 
The elevated GSTs have been found to bind to molecules of various pyrethroid insecticides compromising effectiveness and toxicity by a sequestering mechanism. Although the spread of pyrethroid resistance has increased in disease vectors worldwide, the actual operational impact of resistance in control of disease vectors and transmission remains limited.

Though the results suggest differential involvement of metabolic detoxification, esterases, glutathione-Stransferases and AChE inhibition in the development of acetamiprid resistance in Ae. aegypti; yet increase in insecticide resistance is a multidimensional and dynamic process that depends upon many factors. The increase in the frequency of resistant Ae. aegypti population demands the use of insecticides with different modes of action.

\section{CONCLUSION}

In the present study, susceptible population of Ae. aegypti L. was subjected to acetamiprid selection pressure and resistance was developed. The results suggest the probable role of metabolic detoxifying enzymes, such as elevated esterase levels and glutathione-S-transferases in the development of resistance. The results also indicate individual or synergistic contribution of different enzymes leading to acetamiprid detoxification. However, increase in insecticide resistance is a multifaceted and vigorous process and depends upon many factors. The increase in the frequency of resistant Ae. aegypti population demands the proper insecticide resistance management. The investigations could help in formulation of resistance management strategies to eliminate mosquito as well as in development of new insecticides from the already existing one.

\section{ACKNOWLEDGMENT}

The authors are thankful to Council of Scientific and Industrial Research (CSIR), New Delhi for providing financial assistance. The authors extend thanks to Principal, Acharya Narendra Dev College for providing laboratory and culture facilities to conduct the experiments.

\section{REFERENCES}

[1] J.A. Suaya, D.S. Shepard, M.S. Chang, M. Caram, S. Hoyer, D. Socheat, N. Chantha, M.B. Nathan, Cost-effectiveness of annual targeted larviciding campaigns in Cambodia against the dengue vector Aedes aegypti, Trop. Med. Int. Hlth. 12(9) (2007) 1026-1036. DOI: $10.1111 / \mathrm{j} .1365-$ 3156.2007.01889.x
[2] M.E. Beatty, E. Hunsperger, E. Long, J. Schürch, S. Jain, R. Colindres, G.Lerebours, Y.M. Bernard, J.G. Dobbins, M. Brown, G.G. Clark, Mosquito borne infections after Hurricane Jeanne, Haiti, 2004, Emerg. Infect. Dis. 13(2) (2007) 308-310. DOI: $10.3201 /$ eid1302.061134

[3] T. Chareonviriyaphap, B. Aum-Aung, S. Ratanatham, Current insecticide resistance patterns in mosquito vectors in Thailand, Southeast Asian J. Trop. Med. Publ. Hlth. 30 (1999) 184-194.

[4] T. Chareonviriyaphap, A. Prabaripai, S. Sungvornyothrin, An improved excito-repellency test chamber for mosquito behavioral tests, J. Vector. Ecol. 27 (2002) 250-252.

[5] W.G. Brogdon, J.C. McAllister, Simplification of adult mosquito bioassays through use of timemortality determinations in glass bottles, J. Amer. Mosq. Cont. Assoc. 14(2) (1998) 159-164.

[6] M. Tomizawa, J.E. Casida, Neonicotinoid insecticide toxicology: mechanisms of selective action, Annu. Rev. Pharmacol. Toxicol. 45 (2005) 247-268.

DOI:

10.1146/annurev.pharmtox.45.120403.095930

[7] J.E. Casida, K.A. Durkin, Neuroactive insecticides: targets, selectivity, resistance, and secondary effects, Annu. Rev. Entomol. 58 (2013) 99-117. DOI: 10.1146/annurev-ento-120811153645

[8] N. Simon-delso, V. Amaral-raga, C.P. Belzunes, J.M. Bonmatin, M. Chagnon, C. Downs, L. Furlan, D.W. Gibbons, C. Giorio, V. Girolami, D. Goulson, D.P. Kreutzweiser, C.H. Krupke, M. Liess, E. Long, M. McField, P. Mineau, E.A. Mitchell, C.A. Morrissey, D.A. Noome, L. Pisa, J. Settle, J.D. Stark, A. Tapparo, H. Van Dyck, J. Van Praagh, J.P. Van der Sluijis, P.R. Whitehorn, M. Wiemers, Systemic insecticides (neonicotinoids and fipronil): Trends, uses, mode of action and metabolites, Environ. Sci. Pollut. Res. 22 (2015) 5-34.

[9] R. Schmuck, Ecotoxicological profile of the insecticide thiacloprid, Pflanzenschutz Nachrichten-Bayer-English Edition, 54 (2001) 161-184.

[10] A. Elbert, R. Nauen, W. Leicht, Imidacloprid, a novel chloronicotinyl insecticide: Biological activity and agricultural importance. In: Insecticides with novel modes of action, Springer, Berlin, Heidelberg (1998), pp. 50-73. https://doi.org/10.1007/978-3-662-03565-8_4 
[11] J.E. Casida, G.B. Quistad, Why insecticides are more toxic to insects than people: the unique toxicology of insects. J. Pestic. Sci. 29(2) (2004) 81-86.

[12] US Environmental Protection Agency (USEPA). 2002.

https://www3.epa.gov/pesticides/chem_search/re g_actions/registration/fs_PC-099050_15-Mar02.pdf . pp23

[13] A.S. Gunasekara, T. Truong, K.S. Goh, F. Spurlock, R.S. Tjeerdema, Environmental fate and toxicology of fipronil, J. Pestic. Sci. 32 (2007)189-

199. https://doi.org/10.1584/jpestics.R07-02

[14] D. Goulson, An overview of the environmental risks posed by neonicotinoid insecticides. J. App. Ecol. 50(4) (2013) 977-987. https://doi.org/10.1111/1365-2664.12111

[15] A. Elbert, M. Haas, B. Springer, W. Thielert, R. Nauen, Applied aspects of neonicotinoid uses in crop protection, Pest Manag. Sci. 64(11) (2008) 1099-1105. DOI: 10.1002/ps.1616

[16] A. Sympathy, B. Rai Crop Protection Division, Indian Institute of Vegetable Research Varanasi. (2006) 1 .

[17] J. Kimura-Kuroda, Y . Komuta, Y . Kuroda, M . Hayashi, H. Kawano, Nicotine-like effects of the neonicotinoid insecticides acetamiprid and imidacloprid on cerebellar neurons from neonatal rats, (2012) PLoS One. 7p.e32432. https://doi.org/10.1371/journal.pone.0032432

[18] M.O. Jian-chu, Y. Tian-ci, C. Jia-an, S. Xiaogang, Lethal and sublethal effects of acetamiprid on the larvae of Culex pipiens pallens, Insect Sci. 9(3) (2002) 45-49.

[19] F.P. Sanchez-Bayo, Insecticides mode of action in relation to their toxicity to non-target organisms, J. Environ. Anal. Toxicol. 4 (2012) 1-11. DOI:10.417 2/2161-0525.S4-002

[20] H.J. Takahashi, M. Mitsui, M. Yano, T. Take, T. Yamada. Efficacy of acetamiprid 2\% granule against diamondback moth on cabbage by various application methods, J. Pestic. Sci. 24 (1999) 2327.

[21] R. Warikoo, A. Ray, J.K. Sandhu, R. Samal, N. Wahab, S. Kumar, Larvicidal and irritant activities of hexane leaf extracts of Citrus sinensis against dengue vector Aedes aegypti L., Asian Pac. J. Trop. Biomed. 2(2) (2012) 152-155. DOI: $10.1016 / \mathrm{S} 2221-1691(11) 60211-6$
[22] S. Kumar, M. Mishra, N. Wahab, R. Warikoo, Larvicidal, repellent, and irritant potential of the seed-derived essential oil of Apium graveolens against dengue vector, Aedes aegypti L. (Diptera: Culicidae), Front. Publ. Hlth. 2 (2014) 147, 1-6. DOI: $10.3389 /$ fpubh.2014.00147

[23] R.R. Samal, S. Kumar, Susceptibility status of Aedes aegypti L. against different classes of insecticides in New Delhi, India to formulate mosquito control strategy in fields, Open Parasitol. J. 6(1) (2018) 52-62. DOI: $10.2174 / 1874421401806010052$

[24] World Health Organization. Techniques to detect insecticide resistance mechanisms (field and laboratory manual). World Health Organization (1998). apps.who.int/iris/handle/10665/83780

[25] M.R. Bonner, J. Coble, A. Blair, L.E. Beane Freeman, J.A. Hoppin, D.P. Sandler, M.C. Alavanja, Malathion exposure and the incidence of cancer in the agricultural health study, Am. J. Epidemiol. 166(9) (2007) 1023-1034. DOI: $10.1093 / \mathrm{aje} / \mathrm{kwm} 182$

[26] N. Liu, Insecticide resistance in mosquitoes: Impact, mechanisms, and research directions, Annu. Rev. Entomol. 60 (2015) 537559. DOI: 10.1146/ annurev-ento-010814-020828

[27] J. Hemingway, L. Field, J. Vontas, An overview of insecticide resistance, Science, 298(5591) (2002) 96-97. DOI: 10.1126/science.1078052

[28] S. Uragayala, V.Verma, E. Natarajan, P.S. Velamuri, R. Kamaraju, (2015). Adulticidal \& larvicidal efficacy of three neonicotinoids against insecticide susceptible \& resistant mosquito strains, Ind. J. Med. Res. 142(2015) S64-S70. DOI: $10.4103 / 0971-5916.176624$

[29] D.K. Nomura, K.A. Durkin, K.P. Chiang, G.B. Quistad, B.F. Cravatt, J.E. Casida, Serine hydrolase KIAA1363: toxicological and structural features with emphasis on organophosphate interactions, Chem. Res. Toxicol, 19(9) (2006) 1142-1150. DOI: $10.1021 /$ tx060117m

[30] S. Bhan, L. Mohan, C.N. Srivastava, Relative larvicidal potentiality of nano-encapsulated temephos and imidacloprid against Culex quinquefasciatus, J. Asia-Pacific Entomol. 17(4) (2014)

787-791.

DOI: 10.1016/j.aspen.2014.07.006

[31] N.H.Z. Safi, A.A. Ahmadi, S. Nahzat, S.P. Ziapour, S.H. Nikookar, M. Fazeli-Dinan, A. Enayati, J. Hemingway, Evidence of metabolic 
nomenclature, Pharmacogenetics 6(1) (1996) 1-

mechanisms playing a role in multiple insecticides resistance in Anopheles stephensi populations from Afghanistan, Malar. J. 16(1) (2017) 100-134. DOI: 10.1186/s12936-017-1744-9

[32] O. Wan-Norafikah, W.A. Nazni, H.L. Lee, P. Zainol-Ariffin, M. Sofian-Azirun, (2010). Permethrin resistance in Aedes aegypti (Linnaeus) collected from Kuala Lumpur, Malaysia, J. AsiaPacific Entomol. 13(3) (2010) 175-182. DOI: $10.1016 /$ j.aspen.2010.03.003

[33] D.R. Nelson, L. Koymans, T. Kamataki, J.J. Stegeman, R. Feyereisen, D.J. Waxman, M.R. Waterman, O. Gotoh, M.J. Coon, R.W. Estabrook, I.C. Gunsalus, P450 superfamily: update on new sequences, gene mapping, accession numbers and
42. DOI: 10.1089/dna.1993.12.1

[34] D.R. Roberts, R.G. Andre, Insecticide resistance issues in vector-borne disease control, Am. J. Trop. Med. Hyg. 50(6) (1994) 21-34. DOI: $10.4269 /$ ajtmh.1994.50.21

[35] R. Feyereisen, Insect P450 enzymes, Annu. Rev. Entomol. 44(1) (1999) 507-533. DOI: 10.1146/ann urev.ento.44.1.507

[36] P. Somboon, L.A. Prapanthadara, W. Suwonkerd, Insecticide susceptibility tests of Anopheles minimus sl, Aedes aegypti, Aedes albopictus, and Culex quinquefasciatus in Northern Thailand, Southeast Asian J. Trop. Med. Publ. Hlth. 34(1) (2003) 87-93. 